\title{
Presence of Chronic Obstructive Pulmonary Disease (COPD) Impair Survival in Lung Cancer Patients Receiving Epidermal Growth Factor Receptor-Tyrosine Kinase Inhibitor (EGFR-TKI): A Nationwide, Population-Based Cohort Study
}

\author{
Chia-Che Wu ${ }^{1,+}{ }^{+}$, Kun-Ming Rau ${ }^{2,+}$, Wei-Chieh Lee ${ }^{3}\left(\mathbb{D}\right.$, Meng-Che Hsieh ${ }^{2}$, Jia-Sin Liu ${ }^{4}{ }^{\oplus}$, \\ Yen-Yang Chen ${ }^{1}$ and Harvey Yu-Li Su ${ }^{1,5, * \mathbb{D}}$ \\ 1 Division of Hematology Oncology, Department of Internal Medicine, \\ Kaohsiung Chang Gung Memorial Hospital and Chang Gung University, College of Medicine, \\ Kaohsiung 83301, Taiwan \\ 2 Department of Hematology Oncology, E-Da Cancer Hospital and I-Shou University, College of Medicine, \\ Kaohsiung 82445, Taiwan \\ 3 Division of Cardiology, Department of Internal Medicine, Kaohsiung Chang Gung Memorial Hospital and \\ Chang Gung University, College of Medicine, Kaohsiung 83301, Taiwan \\ 4 Department of Public Health, College of Health Science, Kaohsiung Medical University, Kaohsiung, \\ Kaohsiung 80708, Taiwan \\ 5 Clinical Trial Center, Kaohsiung Chang Gung Memorial Hospital, Kaohsiung 83301, Taiwan \\ * Correspondence: yolisu@mac.com; Tel.: +886-7-731-7123 (ext. 8303); Fax: +886-7-732-2402 \\ + C.-C.W. and K.-M.R. contributed equally to the study.
}

Received: 3 June 2019; Accepted: 10 July 2019; Published: 12 July 2019

check for updates

\begin{abstract}
The emergence of epidermal growth factor receptor-tyrosine kinase inhibitor (EGFR-TKI) caused a paradigm shift in the treatment of non-small cell lung cancer (NSCLC). Although several clinicopathologic factors to predict the response to and survival on EGFR-TKI were recognized, its efficacy has not been confirmed for patients with underlying pulmonary disease, such as chronic obstructive pulmonary disease (COPD). We conducted the study to evaluate the impact of COPD on survival for NSCLC patients that underwent EGFR-TKI treatment. The nationwide study obtained clinicopathologic data from the National Health Insurance Research Database in Taiwan between 1995 and 2013. Patients receiving EGRR-TKI were divided into COPD and non-COPD groups, and adjusted for age, sex, comorbidities, premium level and cancer treatments. Overall survival (OS) and progression-free survival (PFS) were calculated by Kaplan-Meier analysis. In total, 21,026 NSCLC patients were enrolled, of which $47.6 \%$ had COPD. After propensity score (PS) matching, all covariates were adjusted and balanced except for age $(p<0.001)$. In the survival analysis, the median OS ( 2.04 vs. 2.28 years, $p<0.001)$ and PFS $(0.62$ vs. 0.69 years, $p<0.001)$ of lung cancer with COPD were significantly worse than those without COPD. Lung cancer patients on EGFR-TKI treatment had a worse survival outcome if patients had pre-existing COPD.
\end{abstract}

Keywords: COPD; EGFR-TKI; lung cancer; survival; Taiwan

\section{Introduction}

Lung cancer is a global common malignancy, and accounts for the leading cause of cancer-related death in the USA, Asia and Taiwan [1,2]. Although much progress in treatment of metastatic non-small cell lung cancer (NSCLC) has been made in recent decades, the 5-year survival of NSCLC in Taiwan 
remains poor (all stages, 16.3\%). With increasing knowledge about oncogenic mutation of NSCLC, several effective therapeutic opinions have been developed, including a specific antibody to the epidermal growth factor receptor (EGFR) and anaplastic lymphoma kinase (ALK). For NSCLC harboring EGFR driver mutation, the current standard of treatment in the first-line setting is EGFR tyrosine kinase inhibitor (TKI), either first-generation (gefitinib or erlotinib) or second-generation TKI (afatinib) [3-6]. A recent study, analyzing six randomized clinical trials, compared EGFR-TKI with conventional chemotherapy in the first-line setting and showed a significant increment in progressive-free survival (PFS) from 5.6 months with chemotherapy to 11.0 months with EGFR-TKI [7]. Although the presence of activating EGFR mutation (del19 or L858R) precisely predicts good response to EGFR-TKI, there are, however, several clinical factors showing various responses to EGFR-TKI, and the underlying mechanism still remains unknown [8].

Lung cancer patients are generally older, and beyond all doubt, associated with high prevalence of coexisting comorbidity such as chronic obstructive pulmonary disease (COPD), congestive heart failure, diabetes mellitus and cardiovascular disease [9]. COPD is considered as a cigarette-related chronic pulmonary inflammatory disease, which shares a common pathogenic pathway with lung cancer. Several studies showed COPD increased the risk for NSCLC, notably independent to patient age or extent of cigarette exposure [10-12]. Moreover, the relationship between COPD and overall survival of NSCLC has drawn much research interests, although the result still remains inconclusive. A recent meta-analysis, consisting of 26 qualified observational studies, showed that presence of COPD highly correlated with poor survival in localized and resected NSCLC [13]. However, among patients that received chemotherapy for metastatic NSCLC, several studies showed inconclusive results in discussing the survival impact of COPD in metastatic NSCLC [14,15]. Also, there is not enough evidence to explore the role of COPD in patients treated with EGFR-TKI. Therefore, we conducted a large-scale, nationwide, population-based study by using the National Health Insurance Research Database (NHIRD) to investigate the prognostic role of COPD in metastatic NSCLC patients receiving EGFR-TKI.

\section{Material and Methods}

\subsection{Data Source and Study Population}

In the population-based cohort study, we used the Taiwan National Health Insurance Research Database (NHIRD) as the data source. The National Health Insurance (NHI) program was initiated in 1995, providing universal and compulsory health care that covers $>99 \%$ of all residents $(23.7$ million people) in Taiwan. The NHIRD consists of unrecognizable longitudinal health care claims data, including sex, date of birth, medical institutions, medical or surgical procedures, and all information regarding inpatient and outpatient visits. The diagnoses were coded in accordance with the International Classification of Diseases, Ninth Revision, Clinical Modification (ICD-9-CM). The Bureau of NHI requires registration of patients with "catastrophic illness", such as end-stage renal disease, congenital abnormalities, autoimmune diseases and all types of cancers. The vulnerable beneficiaries who were issued with a Catastrophic Illness Certificate (CIC) will be exempted from copayments for the corresponding medical services. Approval of CIC requires strict evaluation by experts in the Bureau of the NHI. The NHIRD encrypts all recognizable information of beneficiaries, and thus provides investigators anonymous data to analyze, with strict confidential guidelines.

\subsection{Study Cohort}

We identified all lung cancer patients (ICD-9-CM: 162) using either hospitalization or outpatient diagnoses between 1995 and 2013. The date of lung cancer diagnosis, either by clinical imaging or pathologic confirmation, was set as the index date. To explore our hypothesis, we confined study cohort to lung cancer patients that underwent EGFR-TKI (gefitinib or erlotinib) and divided it into two subgroups based on the presence of COPD (ICD-9-CM: 491, 492, 496). We further performed propensity score matching to balance the intergroup difference of covariates. 
In Taiwan, the application and use of EGFR-TKI for third-line therapy of chemotherapy refractory NSCLC by NHI reimbursement has been approved since November 2004 (gefitinib). In November 2013, gefitinib and erlotinib got approved and reimbursed as first-line therapy for lung adenocarcinoma harboring active EGFR mutations. Physicians must evaluate the treatment response of EGFR-TKI every three months by imaging tools. Once progressive disease was documented by imaging, re-application of EGFR-TKI was not allowed and be turned down by the NHI.

\subsection{Demographic Covariates and Comorbidities}

The demographic characteristics of age, sex, income for insurance payment and comorbidities using the Charlson comorbidity index (CCI), which included congestive heart failure (CHF), diabetes mellitus (DM), pneumonia and sepsis, were identified and considered as covariates. Patients were classified in three levels of monthly income: NTD $<15,840$, NTD 15,841-25,000, and NTD $>25,000$. Anti-cancer treatments, including all kinds of chemotherapy (CT) regimens, radiotherapy (RT) and EGFR-TKI, were also included the study. EGFR-TKI and chemotherapy can be used alternatively, and we also showed the treatment sequence prior to EGFR-TKI. Concomitant drugs, including statins, non-steroidal anti-inflammatory drugs (NSAID), aspirin, anti-hypertension and steroids, were also listed and compared between the two cohorts.

\subsection{Propensity Score Matching}

We performed propensity score matching to eliminate intergroup selection bias. The propensity score (PS) is defined as the conditional probability of lung cancer patients with or without COPD given in some measurable covariates. A non-parsimonious multivariable logistic regression model was used for calculating PS. The covariates in the 1:1 PS matching model were adjusted for age, gender, monthly income, various comorbidities and many types of chemotherapy, radiotherapy and EGFR-TKI treatment.

\subsection{Endpoints}

The main endpoint of the study was overall survival (OS). OS was defined as the time period from the index date to any cause of death or censored at the last follow-up visit. The second endpoint was progression-free survival (PFS), which was defined as the period between administration of EGFR-TKI and the end of dosing. Physicians may discontinue EGFR-TKI treatment based on imaging (CT, MRI or chest X-ray) progression, abnormal physical examination (e.g., enlarged lymph node), remarkedly increased tumor markers or toxicity. Although our study was not designed as a prospective study with a scheduled imaging survey, the PFS could be roughly reliable because of strict scrutiny of EGFR-TKI application.

\subsection{Statistical Analysis}

The intergroup differences in demographic variables, comorbidities and treatments were compared by $t$ tests for continuous variables and by chi-squared tests of Fisher's exact test for categorical variables. We performed survival analysis for OS and PFS by Kaplan-Meier analysis and compared significance by the log-rank test. To calculate the hazard ratio (HR) of the risk of death and progression-free survival, we used Cox proportional hazard regression analysis. An adjusted model was conducted by adjusting age, gender, premium level, comorbidities, anti-cancer treatments and EGFR-TKIs in a multivariate analysis. All analyses were conducted using SAS statistical software analysis (Version 9.4; SAS institute, Cary, NC, USA)

\section{Results}

\subsection{Patient Characteristics}

In total, we screened 153,642 lung cancer patients initially. To identify the EGFR-TKI users, we excluded 9915 individuals due to missing or unclear diagnosis dates, 51,283 individuals due to 
diagnosis dates before EGFR-TKI reimbursement, and 71,417 patients without any records of EGFR-TKI administration. Figure 1 demonstrate the CONSORT diagram and patients flow in detail. At last, we identified a total of 21,026 lung cancer patients treated with EGFR-TKI during the study period. The detailed demographic information of whole EGFR-TKI users is shown in Table 1. Among them, $47.6 \%$ of patients had the diagnosis of COPD, $4 \%$ of congestive heart failure, $19 \%$ of diabetes mellitus, $16 \%$ of pneumonia and $0.9 \%$ of them had the diagnosis of sepsis. For patients receiving EGFR-TKI, $47 \%$ of all were erlotinib, $62 \%$ of them were gefitinib and $9 \%$ of all received both erlotinib and gefitinib. Approximately two thirds of patients (68\%) underwent chemotherapy, and the mostly commonly used regimens were gemcitabine $(41 \%)$, vinorelbine $(38 \%)$, docetaxel $(34 \%)$, pemetrexed $(33 \%)$ and cisplatin (20\%).

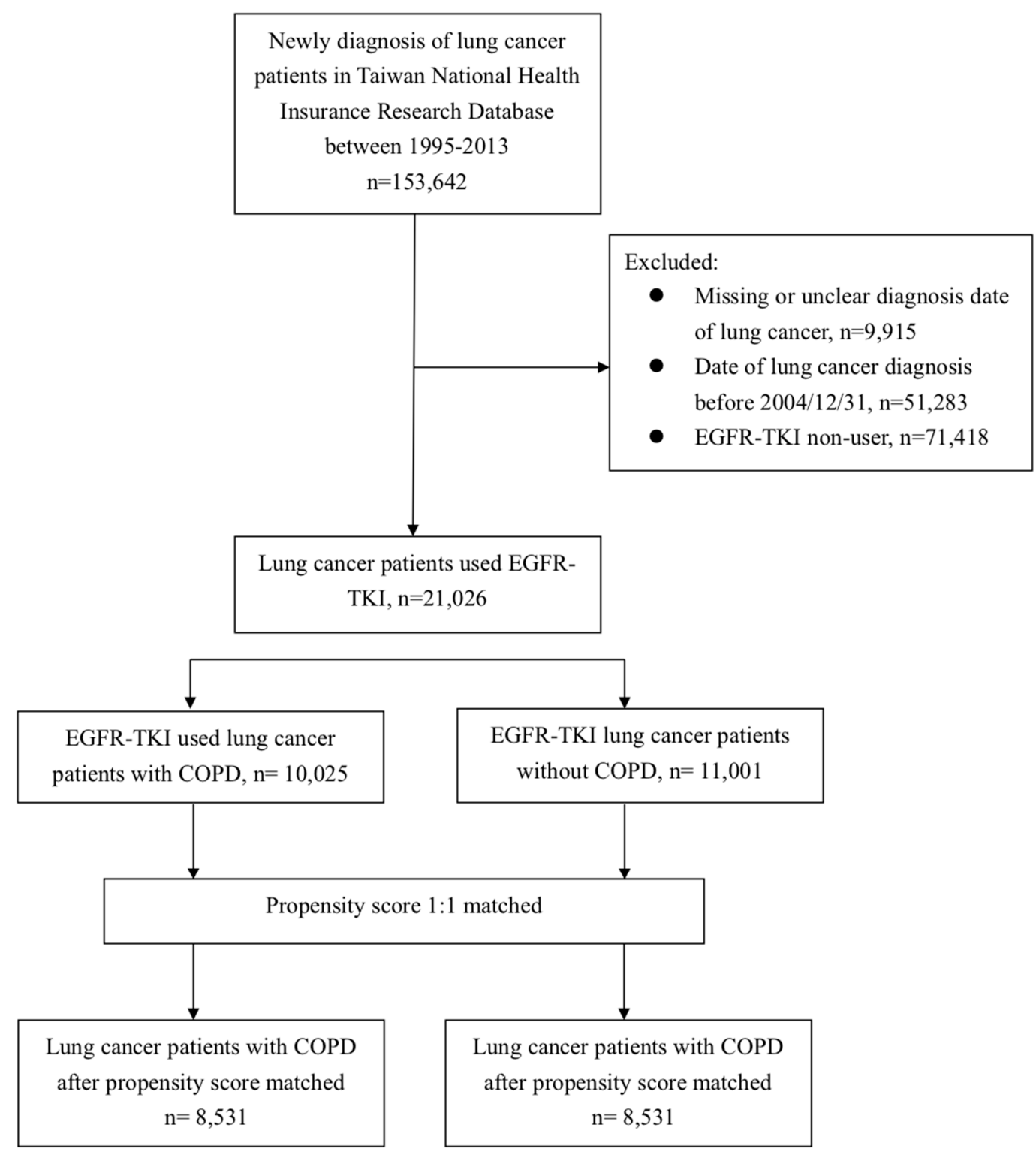

Figure 1. Flow diagram of patient's enrollment.

In the model before PS matching, there were statistically significant differences between COPD and non-COPD groups, including age $(p<0.001)$, sex $(p<0.001)$, premium level $(p<0.001)$, comorbidities $(p<0.001)$, anti-neoplastic agents $(p<0.001)$ and concomitant drugs $(p<0.001)$. After 1:1 PS adjustment, there were 8531 individuals allocated equally in both COPD and non-COPD groups. Although the mean age of COPD patients was slightly older than non-COPD patients ( 65.6 vs. 64.9 years, $p<0.001$ ), 
there were no other significant intergroup differences regarding to gender, premium level, comorbidity and use of anti-neoplastic agents (Table 1).

Table 1. The demographic characteristics of lung cancer patients with epidermal growth factor receptortyrosine kinase inhibitor (EGFR-TKI).

\begin{tabular}{|c|c|c|c|c|c|c|}
\hline & \multicolumn{3}{|c|}{ Before Propensity Score Matched } & \multicolumn{3}{|c|}{ After Propensity Score Matched } \\
\hline & With COPD & $\begin{array}{l}\text { Without } \\
\text { COPD }\end{array}$ & $p$ Value & With COPD & $\begin{array}{l}\text { Without } \\
\text { COPD }\end{array}$ & $p$ Value \\
\hline Age (mean, SD), years & $67.6(11.8)$ & $62(12.1)$ & $<0.001$ & $65.6(11.3)$ & $64.9(11.4)$ & $<0.001$ \\
\hline \multicolumn{7}{|l|}{ Gender } \\
\hline Male & $5167(51.5)$ & $4804(43.7)$ & $<0.001$ & $4091(48.0)$ & $4030(47.2)$ & 0.35 \\
\hline$>25,000$ & $3588(35.8)$ & 4934 (44.9) & $<0.001$ & $3373(39.5)$ & $3480(40.8)$ & 0.09 \\
\hline 25000-15840 & $4510(45.0)$ & $4313(39.2)$ & $<0.001$ & 3657 (42.9) & $3567(41.8)$ & 0.16 \\
\hline$<15,840$ & $1179(11.8)$ & $1027(9.3)$ & $<0.001$ & $883(10.4)$ & $858(10.1)$ & 0.53 \\
\hline Low & $748(7.5)$ & $727(6.6)$ & 0.016 & $618(7.2)$ & $626(7.3)$ & 0.81 \\
\hline \multicolumn{7}{|l|}{ Comorbidity } \\
\hline CCI score & $4.7(2.7)$ & $4.2(2.8)$ & $<0.001$ & $4.4(2.6)$ & $4.4(2.9)$ & 0.99 \\
\hline \multicolumn{7}{|l|}{ Anti-cancer agents } \\
\hline Erlotinib & $4770(47.6)$ & $4990(45.4)$ & 0.001 & 4004 (46.9) & $3991(46.8)$ & 0.84 \\
\hline Gefitinib & $6071(60.6)$ & $7083(64.4)$ & $<0.001$ & $5278(61.9)$ & $5302(62.1)$ & 0.71 \\
\hline Erlotinib + Gefitinib & $816(8.1)$ & $1072(9.7)$ & $<0.001$ & $751(8.8)$ & $762(8.9)$ & 0.77 \\
\hline Gemcitabine & $3962(39.5)$ & $4689(42.6)$ & $<0.001$ & 3547 (41.6) & $3546(41.6)$ & 0.99 \\
\hline Docetaxel & $3321(33.1)$ & $3857(35.1)$ & 0.003 & $2965(34.8)$ & $2947(34.5)$ & 0.77 \\
\hline Pemetrexed & $3070(30.6)$ & $3864(35.1)$ & $<0.001$ & $2843(33.3)$ & 2845 (33.3) & 0.99 \\
\hline Vinorelbine & $3894(38.8)$ & $4113(37.4)$ & 0.03 & $3262(38.2)$ & 3219 (37.7) & 0.50 \\
\hline Cisplatin & $1852(18.5)$ & $2487(22.6)$ & $<0.001$ & $1737(20.4)$ & $1764(20.7)$ & 0.61 \\
\hline Paclitaxel & $1256(12.5)$ & $1498(13.6)$ & 0.019 & $1120(13.1)$ & $1136(13.3)$ & 0.72 \\
\hline 0 & $3627(36.2)$ & $3956(36.0)$ & 0.74 & 3013 (35.3) & 3043 (35.7) & 0.63 \\
\hline 1 & $2705(27.0)$ & $2880(26.2)$ & 0.19 & $2271(26.6)$ & $2249(26.4)$ & 0.70 \\
\hline$\geq 2$ & 3693 (36.8) & 4165 (37.9) & 0.13 & 3247 (38.1) & $3239(38.0)$ & 0.90 \\
\hline \multicolumn{7}{|l|}{ Concomitant drug } \\
\hline Statin & $2151(21.5)$ & $1765(16.0)$ & $<0.001$ & $1692(19.8)$ & $1629(19.1)$ & 0.22 \\
\hline NSAID & $9480(94.6)$ & $9964(90.6)$ & $<0.001$ & $8000(93.8)$ & 7956 (93.3) & 0.17 \\
\hline Aspirin & $3202(31.9)$ & $2332(21.2)$ & $<0.001$ & $2304(27.0)$ & $2215(26.0)$ & 0.12 \\
\hline Anti-HTN & 7265 (72.5) & $6403(58.2)$ & $<0.001$ & $5832(68.4)$ & $5690(66.7)$ & 0.02 \\
\hline Steroids & $7820(78.0)$ & $8029(73.0)$ & $<0.001$ & 6509 (76.3) & $6462(75.7)$ & 0.40 \\
\hline Propensity score & $\begin{array}{c}0.6236 \\
(0.4845)\end{array}$ & $\begin{array}{c}0.5379 \\
(0.4986)\end{array}$ & $<0.001$ & $\begin{array}{c}0.4859 \\
(0.1201)\end{array}$ & $\begin{array}{c}0.4778 \\
(0.1266)\end{array}$ & $<0.001$ \\
\hline
\end{tabular}

Abbreviations: EGFR, epidermal growth factor receptor; TKI, tyrosine kinase inhibitor; SD, standard deviation; COPD, chronic obstructive pulmonary disease; NTD, new Taiwan dollar; CHF, congestive heart failure; DM, diabetes mellitus; $\mathrm{CCI}$, Charlson comorbidity index; $\mathrm{CT}$, chemotherapy; RT, radiotherapy; NSAID, non-steroidal anti-inflammatory drug; and HTN, hypertension.

\subsection{PFS and OS in COPD and non-COPD EGFR-TKI Users}

With a median follow-up of 2.1 years (standard deviation (SD), 1.6 years), the median PFS on EGFR-TKI calculated by Kaplan-Meier analysis in the COPD cohort (before PS matching) was 0.62 years, significantly shorter than the non-COPD cohort ( 0.69 years, $p<0.001$; Figure 2$)$. Of note, non-COPD lung cancer patients had a significantly longer median OS than the COPD cohort (2.28 vs. 2.04 years, $p<0.001$; Figure 3). We also estimated the PFS and OS difference in the model after PS matching. 
Unsurprisingly, the COPD cohort had a significantly worse PFS ( 0.43 vs. 0.47 years; $p=0.032$; Figure 4 ) and OS (1.71 vs. 1.75 years, $p=0.005$; Figure 5$)$ compared to the non-COPD cohort.

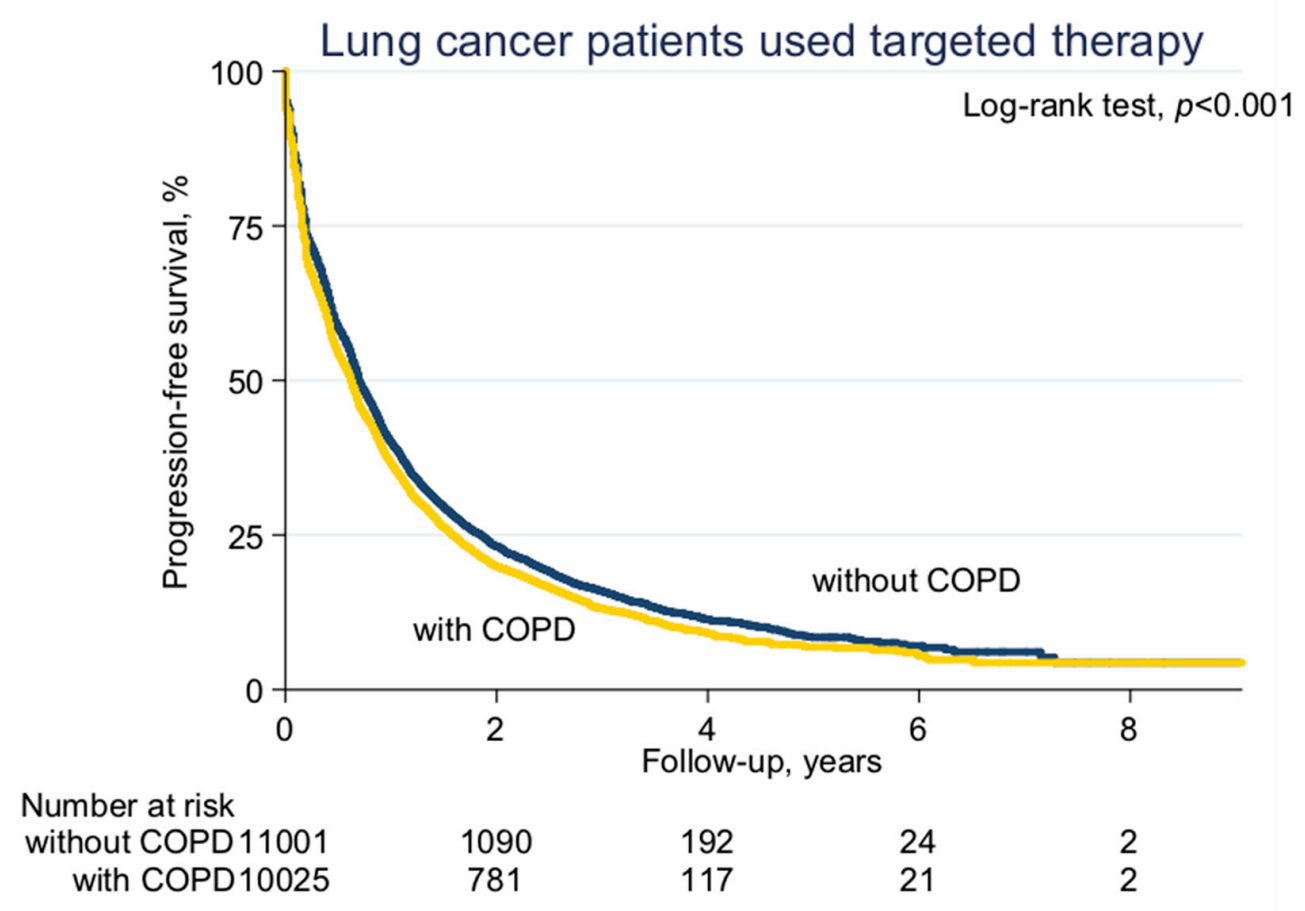

Figure 2. Progression-free survival curve of chronic obstructive pulmonary disease (COPD) and non-COPD cohorts (before propensity score (PS) matching).

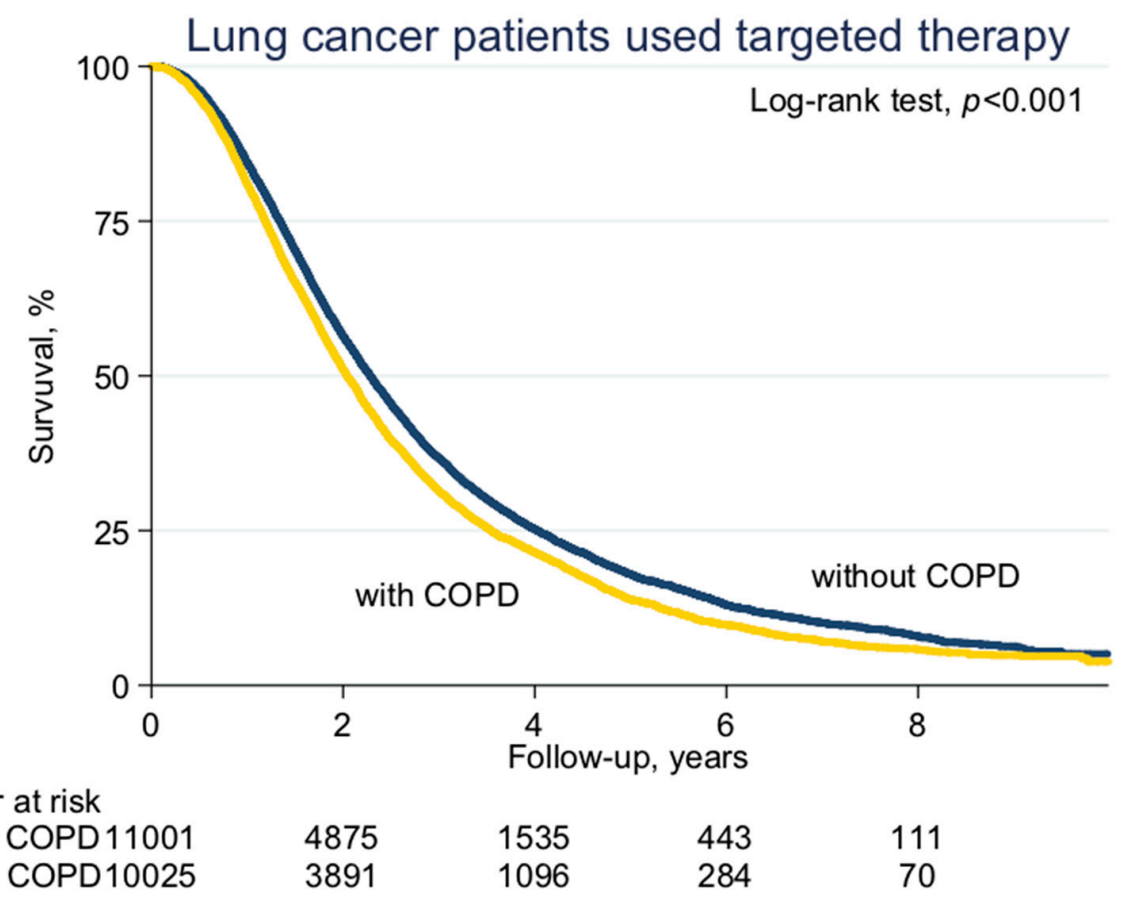

Figure 3. Overall survival curve of COPD and non-COPD cohorts (before PS matching). 


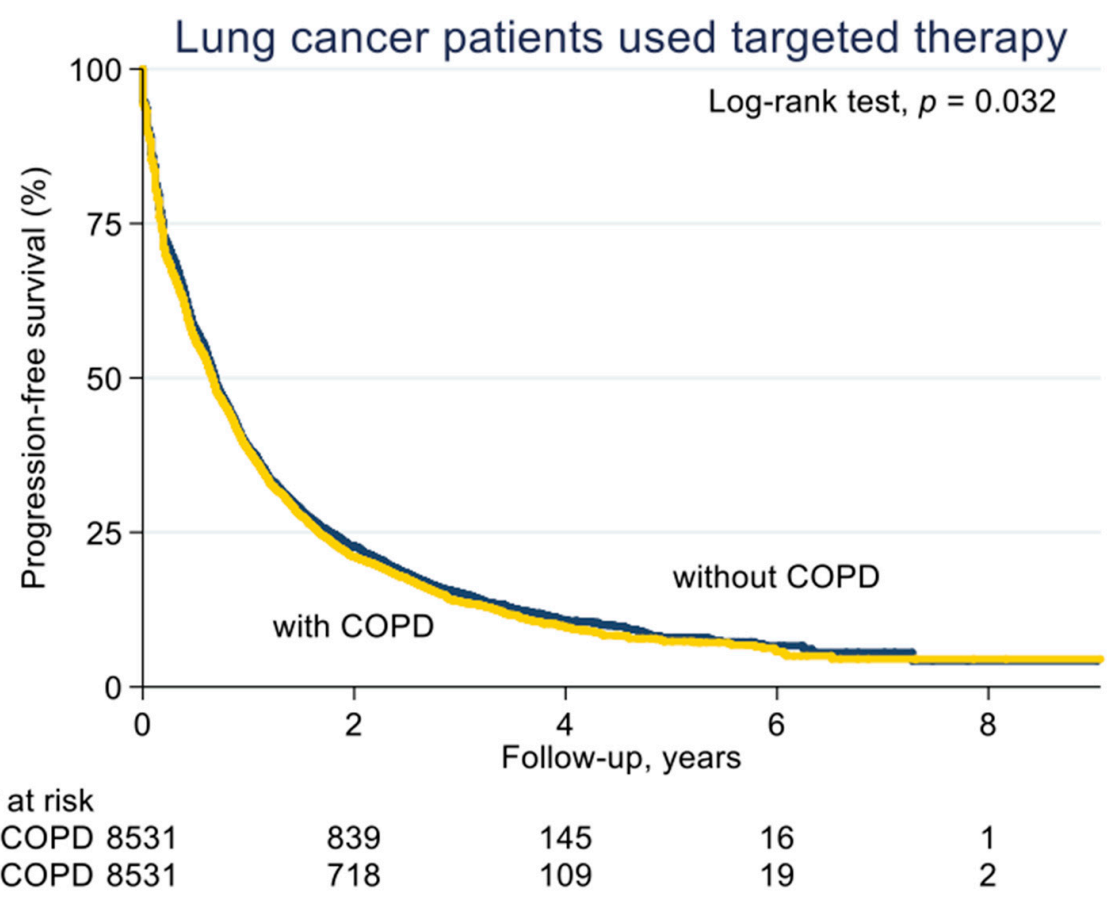

Figure 4. Progression-free survival curve of COPD and non-COPD cohorts (after PS matching).

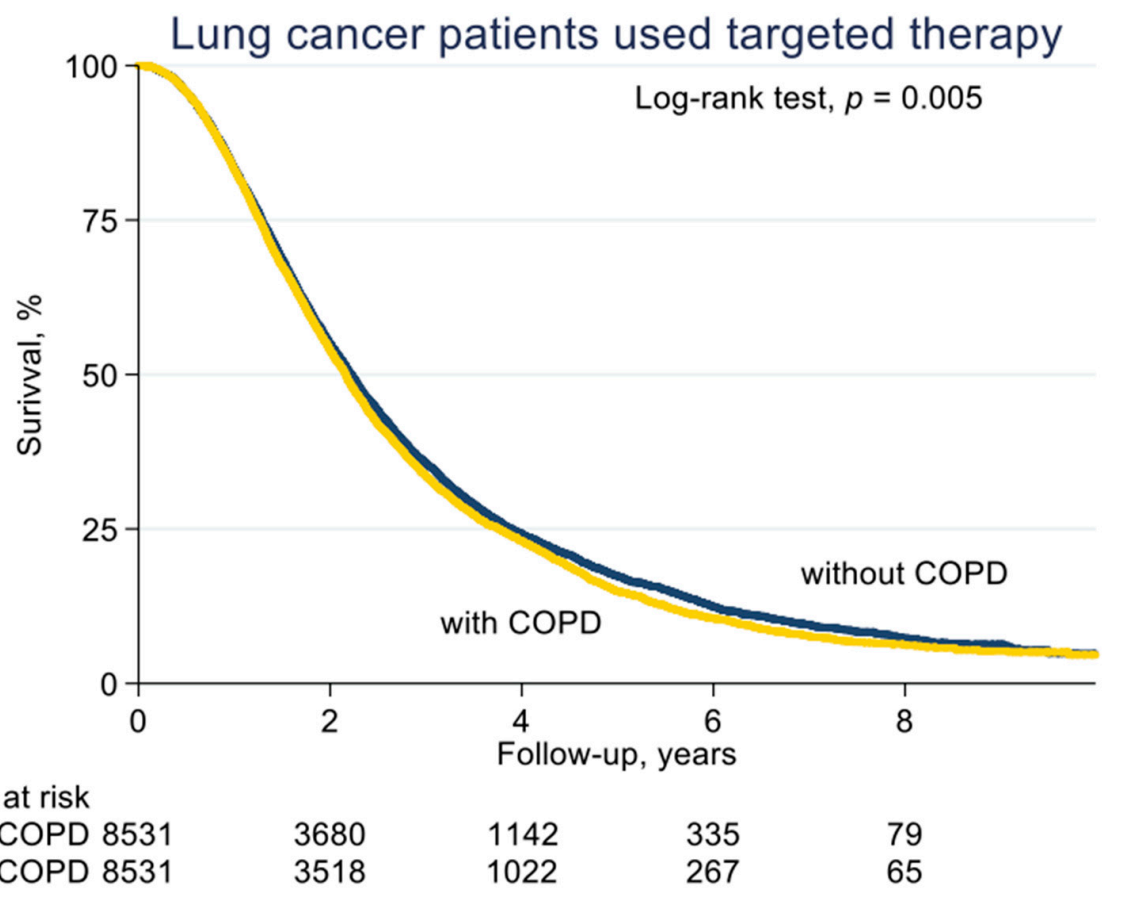

Figure 5. Overall survival curve of COPD and non-COPD cohorts (after PS matching).

\subsection{Comparison of HRs of Death for All Covariates}

Table 2 shows the risk of death in COPD and non-COPD cohorts comparing all clinical covariates. Being male (HR 1.33, 95\% CI 1.29-1.38, $p<0.001$ ), a low premium level of $<15,840$ NTD (HR 1.22, 95\% CI 1.16-1.30, $p<0.001$ ), CHF (HR 1.16, 95\% CI 1.08-1.26, $p<0.001$ ), DM (HR 1.12, 95\% CI 1.07-1.17, $p<0.001$ ), pneumonia (HR 1.25, 95\% CI 1.19-1.30, $p<0.001$ ), sepsis (HR 1.44, 95\% CI 1.22-1.70, $p<0.001$ ) and NSAID (HR 1.18, 95\% CI 1.10-1.27, $p<0.001$ ) were the significant risks of death in the crude univariate analysis. Patients that underwent EGFR-TKI, radiotherapy (RT) or chemotherapy (CT), irrespective of any regimen, had significantly reduced risks of death. Concomitant medications 
such as statin (HR 0.86, 95\% CI 0.83-0.90, $p<0.001$ ), aspirin (HR 0.94, 95\% CI 0.91-0.98, $p<0.001$ ) and anti-hypertension agents (HR 1.33, 95\% CI 1.29-1.38, $p<0.001$ ) were significantly associated with a reduction in mortality.

Table 2. Comparison of the hazard ratios (HRs) of death for all clinical variables.

\begin{tabular}{|c|c|c|c|c|c|c|}
\hline & \multicolumn{2}{|c|}{ Crude Model } & \multicolumn{2}{|c|}{ Adjusted Model $^{\text {a }}$} & \multicolumn{2}{|c|}{$\begin{array}{c}\text { Propensity Score } \\
\text { Matched Adjusted Model }\end{array}$} \\
\hline & $\operatorname{HR}(95 \% \mathrm{CI})$ & $p$ Value & $\operatorname{HR}(95 \% \mathrm{CI})$ & $p$ Value & $\operatorname{HR}(95 \% \mathrm{CI})$ & $p$ Value \\
\hline \multicolumn{7}{|l|}{ All-cause mortality } \\
\hline COPD & $1.16(1.12-1.20)$ & $<0.001$ & $1.05(1.01-1.08)$ & 0.012 & $1.04(1.00-1.08)$ & 0.033 \\
\hline Erlotinib & $0.87(0.84-0.90)$ & $<0.001$ & $0.56(0.53-0.60)$ & $<0.001$ & $0.56(0.52-0.60)$ & $<0.001$ \\
\hline Gefitinib & $0.87(0.84-0.90)$ & $<0.001$ & $0.55(0.52-0.59)$ & $<0.001$ & $0.55(0.51-0.59)$ & $<0.001$ \\
\hline \multicolumn{7}{|l|}{ Gender } \\
\hline Male vs. Female & $1.33(1.29-1.38)$ & $<0.001$ & $1.33(1.29-1.38)$ & $<0.001$ & $1.32(1.27-1.38)$ & $<0.001$ \\
\hline \multicolumn{7}{|l|}{ Premium level (NTD) } \\
\hline$>25,000$ & 1.0 (reference) & & 1.0 (reference) & & 1.0 (reference) & \\
\hline $25,000-15,840$ & $1.16(1.09-1.24)$ & $<0.001$ & $1.21(1.12-1.30)$ & $<0.001$ & 1.17 (1.09-1.27) & $<0.001$ \\
\hline$<15,840$ & $1.22(1.16-1.30)$ & $<0.001$ & $1.15(1.08-1.22)$ & $<0.001$ & $1.13(1.05-1.21)$ & $<0.001$ \\
\hline Low & $1.20(1.16-1.24)$ & $<0.001$ & $1.20(1.15-1.24)$ & $<0.001$ & $1.18(1.13-1.23)$ & $<0.001$ \\
\hline \multicolumn{7}{|l|}{ Comorbidity } \\
\hline $\mathrm{CHF}$ & $1.16(1.08-1.26)$ & $<0.001$ & $1.10(1.02-1.19)$ & 0.016 & $1.13(1.03-1.25)$ & 0.013 \\
\hline $\mathrm{DM}$ & $1.12(1.07-1.17)$ & $<0.001$ & $1.15(1.10-1.21)$ & $<0.001$ & $1.14(1.09-1.20)$ & $<0.001$ \\
\hline Pneumonia & $1.25(1.19-1.30)$ & $<0.001$ & $1.19(1.14-1.24)$ & $<0.001$ & $1.19(1.13-1.25)$ & $<0.001$ \\
\hline Sepsis & $1.44(1.22-1.70)$ & $<0.001$ & $1.17(0.99-1.38)$ & 0.06 & 1.24 (1.03-1.49) & 0.022 \\
\hline CCI score & 1.05 (1.04-1.05) & $<0.001$ & $1.05(1.04-1.05)$ & $<0.001$ & 1.05 (1.04-1.05) & $<0.001$ \\
\hline \multicolumn{7}{|l|}{ Anti-neoplastic agents } \\
\hline Gemcitabine & $0.94(0.91-0.97)$ & $<0.001$ & $1.01(0.97-1.05)$ & 0.60 & $1.00(0.95-1.04)$ & 0.99 \\
\hline Docetaxel & $0.89(0.86-0.92)$ & $<0.001$ & $0.98(0.94-1.02)$ & 0.26 & $0.98(0.93-1.02)$ & 0.31 \\
\hline Pemetrexed & $0.69(0.67-0.72)$ & $<0.001$ & $0.79(0.76-0.82)$ & $<0.001$ & $0.79(0.75-0.82)$ & $<0.001$ \\
\hline Vinorelbine & $0.84(0.81-0.87)$ & $<0.001$ & $0.85(0.82-0.88)$ & $<0.001$ & $0.83(0.80-0.86)$ & $<0.001$ \\
\hline Cisplatin & $0.86(0.83-0.90)$ & $<0.001$ & $0.93(0.89-0.97)$ & 0.002 & $0.92(0.87-0.97)$ & 0.001 \\
\hline Paclitaxel & $0.82(0.78-0.85)$ & $<0.001$ & $0.87(0.83-0.92)$ & $<0.001$ & $0.89(0.84-0.94)$ & $<0.001$ \\
\hline Carboplatin & $0.85(0.79-0.90)$ & $<0.001$ & $0.88(0.82-0.94)$ & $<0.001$ & $0.88(0.81-0.95)$ & 0.001 \\
\hline \multicolumn{7}{|l|}{ Cancer treatment } \\
\hline CT & $0.79(0.76-0.82)$ & $<0.001$ & $0.88(0.83-0.93)$ & $<0.001$ & $0.90(0.84-0.96)$ & 0.003 \\
\hline RT & $0.93(0.90-0.96)$ & $<0.001$ & $0.96(0.93-1.00)$ & 0.041 & $0.98(0.94-1.02)$ & 0.23 \\
\hline \multicolumn{7}{|c|}{ CT regimens before EGFR-TKI } \\
\hline 0 & 1.0 (reference) & & 1.0 (reference) & & 1.0 (reference) & \\
\hline 1 & $1.04(1.00-1.09)$ & 0.07 & $1.14(1.07-1.20)$ & $<0.001$ & $1.13(1.06-1.20)$ & $<0.001$ \\
\hline$\geq 2$ & $0.96(0.92-1.00)$ & 0.046 & $1.17(1.09-1.25)$ & $<0.001$ & $1.18(1.09-1.27)$ & $<0.001$ \\
\hline \multicolumn{7}{|l|}{ Concomitant drug } \\
\hline Statin & $0.86(0.83-0.90)$ & $<0.001$ & $0.85(0.81-0.90)$ & $<0.001$ & $0.85(0.80-0.89)$ & $<0.001$ \\
\hline NSAID & $1.18(1.10-1.27)$ & $<0.001$ & $1.23(1.14-1.33)$ & $<0.001$ & $1.15(1.06-1.25)$ & 0.001 \\
\hline Aspirin & $0.94(0.91-0.98)$ & 0.003 & $0.86(0.82-0.89)$ & $<0.001$ & $0.85(0.81-0.89)$ & $<0.001$ \\
\hline Anti-HTN & $0.92(0.89-0.96)$ & $<0.001$ & $0.84(0.81-0.88)$ & $<0.001$ & $0.84(0.81-0.88)$ & $<0.001$ \\
\hline Steroid & $1.00(0.96-1.04)$ & 0.99 & $1.07(1.02-1.12)$ & 0.003 & $1.07(1.02-1.12)$ & 0.011 \\
\hline
\end{tabular}

a: Model adjusted age, gender, premium level, comorbidities, anti-neoplastic agents, cancer treatments, CT regimens before EGFR-TKI and concomitant drug. Abbreviations: EGFR, epidermal growth factor receptor; TKI, tyrosine kinase inhibitor; SD, standard deviation; COPD, chronic obstructive pulmonary disease; NTD, new Taiwan dollar; $\mathrm{CHF}$, congestive heart failure; DM, diabetes mellitus; CCI, Charlson comorbidity index; $\mathrm{CT}$, chemotherapy; RT, radiotherapy; NSAID, non-steroidal anti-inflammatory drug; and HTN, hypertension.

In the adjusted model which was modified for age, sex, premium level, comorbidities, anti-neoplastic agents, cancer treatments, CT regimen before EGFR-TKI and concomitant drugs, lung cancer patients with COPD still remained at a higher risk of death (HR 1.05, 95\% CI 1.01-1.08, $p=0.012$ ). Administration of EGFR-TKI in the adjusted model showed decreased all cause of mortality in a greater magnitude than in the crude model (HR 0.56 for erlotinib, 95\% CI 0.53-0.60, $p<0.001$ ). Other independent prognostic factors in the adjusted model were similar to those in crude model.

Lastly, after PS matching and adjustment for all competitive confounding factors, COPD (HR 1.04, 95\% CI 1.00-1.08, $p=0.03$ ), male (HR 1.32, 95\% CI 1.27-1.38, $p<0.001$ ), low premium level (HR 1.18, 95\% CI 1.13-1.23, $p<0.001$ ), CHF (HR 1.13, 95\% CI 1.03-1.25, $p=0.01$ ), DM (HR 1.14, 95\% CI 1.09-1.20, $p<0.001$ ), pneumonia (HR 1.19, 95\% CI 1.13-1.25, $p<0.001$ ), sepsis (HR 1.24, 95\% CI 1.03-1.49, $p=0.02$ ), $>1$ CT regimen before EGFR-TKI (HR 1.13, 95\% CI 1.06-1.20, $p<0.001$ ), NSAID (HR 1.15, 95\% CI 1.06-1.25, $p=0.001$ ) and steroid (HR 1.07, 95\% CI 1.02-1.12, $p=0.01$ ) were significantly associated 
with an increased risk of death. A reduced risk of death was observed in erlotinib (HR 0.56, 95\% CI $0.52-0.60, p<0.001$ ), gefitinib (HR 0.55, 95\% CI 0.51-0.59, $p<0.001$ ), pemetrexed (HR 0.79, 95\% CI $0.75-0.82, p<0.001$ ), vinorelbine (HR $0.83,95 \%$ CI $0.80-0.86, p<0.001$ ), cisplatin (HR $0.92,95 \%$ CI $0.87-0.97, p=0.001$ ), paclitaxel (HR $0.89,95 \%$ CI $0.84-0.94, p<0.001$ ), carboplatin (HR $0.88,95 \%$ CI $0.81-0.95, p=0.001$ ), statin (HR $0.85,95 \%$ CI $0.80-0.89, p<0.001$ ), aspirin (HR 0.85, 95\% CI 0.81-0.89, $p<0.001$ ) and anti-hypertension agents (HR 0.84, 95\% CI 0.81-0.88, $p<0.001$ ).

\subsection{Comparison of HRs of Progression}

Table 3 shows the HRs of progression-free survival in all covariates. In brief, COPD remained a significant impairment of PFS (HR 1.11, 95\% CI 1.05-1.15, $p<0.001$ ) in the unadjusted model and in the PS matching model (HR 1.05, 95\% CI 1.02-1.09, $p=0.006$ ).

Table 3. Comparison of HRs of progression-free survival for all covariates.

\begin{tabular}{|c|c|c|c|c|c|c|}
\hline & \multicolumn{2}{|c|}{ Crude Model } & \multicolumn{2}{|c|}{ Adjusted Model ${ }^{\text {a }}$} & \multicolumn{2}{|c|}{$\begin{array}{c}\text { Propensity Score } \\
\text { Matched Adjusted Model }\end{array}$} \\
\hline & HR (95\% CI) & $p$ Value & HR (95\% CI) & $p$ Value & HR (95\% CI) & $p$ Value \\
\hline \multicolumn{7}{|l|}{ Progression-free survival } \\
\hline COPD & $1.11(1.08-1.15)$ & $<0.001$ & $1.05(1.02-1.09)$ & 0.004 & $1.05(1.02-1.09)$ & 0.006 \\
\hline Erlotinib & $1.11(1.08-1.15)$ & $<0.001$ & $0.36(0.34-0.39)$ & $<0.001$ & $0.36(0.33-0.39)$ & $<0.001$ \\
\hline Gefitinib & $0.54(0.52-0.56)$ & $<0.001$ & $0.28(0.26-0.30)$ & $<0.001$ & $0.29(0.26-0.31)$ & $<0.001$ \\
\hline $\begin{array}{c}\text { Age (mean, SD), years } \\
\text { Gender }\end{array}$ & \multicolumn{5}{|c|}{ Gender } & 0.09 \\
\hline Male vs. Female & $1.50(1.45-1.55)$ & $<0.001$ & $1.36(1.31-1.41)$ & $<0.001$ & $1.35(1.30-1.41)$ & $<0.001$ \\
\hline \multicolumn{7}{|l|}{ Premium level (NTD) } \\
\hline$>25,000$ & 1.0 (reference) & & 1.0 (reference) & & 1.0 (reference) & \\
\hline $25,000-15,840$ & $1.08(1.01-1.15)$ & 0.026 & $1.23(1.15-1.32)$ & $<0.001$ & $1.22(1.13-1.31)$ & $<0.001$ \\
\hline$<15,840$ & $1.21(1.14-1.28)$ & $<0.001$ & $1.23(1.16-1.31)$ & $<0.001$ & $1.21(1.14-1.30)$ & $<0.001$ \\
\hline Low & $1.13(1.09-1.18)$ & $<0.001$ & $1.16(1.12-1.21)$ & $<0.001$ & $1.16(1.11-1.21)$ & $<0.001$ \\
\hline \multicolumn{7}{|l|}{ Comorbidity } \\
\hline $\mathrm{CHF}$ & $1.10(1.02-1.18)$ & 0.019 & $1.12(1.03-1.21)$ & 0.006 & $1.10(1.00-1.22)$ & 0.05 \\
\hline $\mathrm{DM}$ & $1.05(1.01-1.10)$ & 0.02 & $1.11(1.06-1.16)$ & $<0.001$ & $1.10(1.05-1.15)$ & $<0.001$ \\
\hline Pneumonia & $1.21(1.16-1.26)$ & $<0.001$ & $1.15(1.11-1.20)$ & $<0.001$ & $1.16(1.10-1.21)$ & $<0.001$ \\
\hline Sepsis & $1.24(1.05-1.46)$ & 0.011 & $1.10(0.93-1.30)$ & 0.25 & $1.15(0.96-1.39)$ & 0.13 \\
\hline CCI score & $1.02(1.01-1.02)$ & $<0.001$ & $1.02(1.01-1.02)$ & $<0.001$ & $1.02(1.01-1.02)$ & $<0.001$ \\
\hline \multicolumn{7}{|l|}{ Anti-neoplastic agents } \\
\hline Gemcitabine & $1.45(1.40-1.49)$ & $<0.001$ & $1.24(1.19-1.30)$ & $<0.001$ & $1.22(1.17-1.28)$ & $<0.001$ \\
\hline Docetaxel & 1.33 (1.29-1.38) & $<0.001$ & 1.09 (1.05-1.14) & $<0.001$ & $1.08(1.03-1.13)$ & 0.001 \\
\hline Pemetrexed & $0.99(0.96-1.02)$ & 0.54 & $0.96(0.92-1.00)$ & 0.032 & $0.95(0.91-0.99)$ & 0.02 \\
\hline Vinorelbine & $1.24(1.20-1.28)$ & $<0.001$ & $1.11(1.07-1.15)$ & $<0.001$ & 1.09 (1.05-1.14) & $<0.001$ \\
\hline Cisplatin & $1.10(1.06-1.14)$ & $<0.001$ & $0.90(0.86-0.95)$ & $<0.001$ & $0.89(0.84-0.94)$ & $<0.001$ \\
\hline Paclitaxel & $1.22(1.16-1.27)$ & $<0.001$ & $1.07(1.02-1.12)$ & 0.006 & $1.08(1.02-1.14)$ & 0.006 \\
\hline Carboplatin & $1.08(1.01-1.15)$ & 0.029 & $0.92(0.86-0.99)$ & 0.021 & $0.92(0.85-0.99)$ & 0.029 \\
\hline \multicolumn{7}{|l|}{ Cancer treatment } \\
\hline $\mathrm{CT}$ & $1.43(1.38-1.49)$ & $<0.001$ & $1.01(0.95-1.08)$ & 0.74 & $1.04(0.97-1.11)$ & 0.29 \\
\hline RT & $1.13(1.09-1.16)$ & $<0.001$ & $1.03(0.99-1.07)$ & 0.11 & $1.05(1.01-1.09)$ & 0.024 \\
\hline \multicolumn{7}{|c|}{ CT regimens before EGFR-TKI } \\
\hline 0 & 1.0 (reference) & & 1.0 (reference) & & 1.0 (reference) & \\
\hline 1 & $1.65(1.58-1.72)$ & $<0.001$ & $1.24(1.17-1.31)$ & $<0.001$ & $1.22(1.15-1.30)$ & $<0.001$ \\
\hline$\geq 2$ & $1.93(1.85-2.01)$ & $<0.001$ & $1.31(1.22-1.40)$ & $<0.001$ & $1.33(1.23-1.43)$ & $<0.001$ \\
\hline \multicolumn{7}{|l|}{ Concomitant drug } \\
\hline Statin & $0.82(0.79-0.86)$ & $<0.001$ & $0.86(0.82-0.91)$ & $<0.001$ & $0.86(0.82-0.91)$ & $<0.001$ \\
\hline NSAID & $1.41(1.31-1.52)$ & $<0.001$ & $1.32(1.23-1.43)$ & $<0.001$ & $1.22(1.12-1.33)$ & $<0.001$ \\
\hline Aspirin & $0.91(0.87-0.94)$ & $<0.001$ & $0.89(0.85-0.93)$ & $<0.001$ & $0.88(0.84-0.93)$ & $<0.001$ \\
\hline Anti-HTN & $0.95(0.92-0.98)$ & 0.004 & $0.93(0.89-0.96)$ & $<0.001$ & $0.93(0.89-0.97)$ & 0.002 \\
\hline Steroid & $1.28(1.23-1.33)$ & $<0.001$ & $1.06(1.02-1.11)$ & 0.007 & 1.07 (1.02-1.13) & 0.006 \\
\hline
\end{tabular}

${ }^{a}$ : Model adjusted age, gender, premium level, comorbidities, anti-neoplastic agents, cancer treatments, CT regimens before EGFR-TKI and concomitant drug. Abbreviations: EGFR, epidermal growth factor receptor; TKI, tyrosine kinase inhibitor; SD, standard deviation; COPD, chronic obstructive pulmonary disease; NTD, new Taiwan dollar; $\mathrm{CHF}$, congestive heart failure; DM, diabetes mellitus; CCI, Charlson comorbidity index; CT, chemotherapy; RT, radiotherapy; NSAID, non-steroidal anti-inflammatory drug; and HTN, hypertension.

\section{Discussion}

In this large scale, nationwide, population-based cohort study, we demonstrated that COPD is a crucial prognostic factor for lung cancer patients treated with EGFR-TKI. We believe that this is the first study to emphasize the clinical role of COPD in selecting therapeutic EGFR-TKI for NSCLC patients. 
Several anecdotal studies investigated the relationship between COPD and lung cancer. Both diseases share common causative risks and pathologic pathways [16]. The UK General Practice Research Database, which enrolled more than 4 million patients within a 14-year period, showed that the 3-year survival for lung cancer patients with COPD was almost half of the general population (15\% vs. $26 \% ; p<0.01)$ [17]. Other studies reported that the survival of lung cancer with COPD were significantly worse in different situations $[18,19]$. A recent meta-analysis focused on the impact of COPD on post-resection survival of lung cancer patients, showing that patients with COPD had higher post-operation complications (pneumonia and prolonged mechanical ventilation) and poor overall survival [18]. The reason of inferior survival was likely attributed to inevitable deterioration of pulmonary function following lung resection, and COPD patients with known bad pulmonary function had poor tolerance to surgery, leading to an increased rate of post-operation pneumonia and cancer recurrence [20].

In the era of targeting therapy for lung cancer, EGFR-TKI have been proven as the pivotal therapeutic role for NSCLC harboring an active EGFR mutation in terms of response rate, PFS and OS [5-7]. Several studies emphasized that the frequency of EGFR mutation increased with several clinicopathologic factors, including adenocarcinoma, never smoker and being female [21]. As COPD is a well-known smoking-related disease, the incidence of an EGFR mutation in COPD patients with lung cancer might be lower than in non-COPD patients. Lim et al. reported that COPD was independently associated with a lower prevalence of EGFR mutation (odd ratio $0.197 ; 95 \%$ CI $0.065-0.600 ; p<0.004$ ), and the EGFR mutation rate conversely decreased with increased severity of COPD $(p=0.001)$ [22]. Importantly even in non-smoking patients, the EGFR mutation rate in patients with COPD was significantly lower than those without COPD $(p=0.001)$. Another study led by Takeda et al. showed $25 \%$ EGFR mutation in lung cancer without emphysema and $9 \%$ in those with emphysema [23]. As active EGFR mutation is the critical factor to predict the response and survival outcome for patients that underwent EGFR-TKI treatment, it is unsurprisingly that COPD patients might had poor treatment efficacy on EGFR-TKI because of inherited low EGFR mutation frequency [24].

Beyond the EFGR pathway, several alternative pathways between COPD and lung cancer were also discussed. Xiao et al. performed a comprehensive whole-genome and exome sequencing study to evaluate the different mutation in lung adenocarcinoma with and without COPD [25]. Not surprisingly that prevalence of EGFR mutation was higher in patients without COPD. Of note, a novel low density-lipoprotein receptor-related protein 1B (LRP1B) mutation was identified and significantly increased prevalence in lung cancer with COPD. The $L R P 1 B$ gene is a putative tumor suppressor gene encoding an endocytic LDL-family receptor [26]. Some studies reported that deregulations of the $L R P 1 B$ gene are associated with resistance to chemotherapy and worse survival in cancer $[27,28]$. Other studies discovered several mechanisms mediating lung cancer development in patients with COPD, such as telomere shortening [29,30], oxidative stress [31] and chronic inflammation [32]. Although increasing understandings of carcinogenesis and genomic pathways of COPD lung cancer have been made, there still no available targeting therapy directly to treat these devastated patients.

Our study had some limitations. First, and the most important, the retrospective cohort study lacked evidence of EGFR mutation, which might cause an imbalanced distribution between COPD and non-COPD groups. The bias could be minimized by the high prevalence of EGFR mutation in Taiwan and the administrative process for first-line EGFR-TKI treatment by the NHI. The prevalence of EGFR mutation in Taiwan was much higher $(40.8 \%$ to $62.1 \%$ ) than that in Western countries, indicating that more than half of all lung cancers in Taiwan should be effectively treated with EGFR-TKI [33-35]. In addition, application for first-line EGFR-TKI treatment required attachment of EGFR mutation reports for administrative review by the NHI policy. In our study, $35 \%$ of all enrolled patients underwent first-line EGFR-TKI treatment in both cohorts, indicating that at least one third of patients had documented EGFR mutation and were allocated equally. Second, although the severity of COPD could be identified, it was not correlated with other comorbidities and survival. Despite this, we adjusted all possible confounding factors and performed PS matching, but the difference of age between the two cohorts still could not be eliminated and could therefore be a confounder. 
In conclusion, our study highlighted that lung cancer patients with COPD had a poorer survival outcome than those without COPD when treated with EGFR-TKI. Further research, either prospective randomized controlled trials or basic molecular studies, are needed to validate this finding.

Author Contributions: Conceptualization, K.-M.R.; data curation, M.-C.H.; formal analysis, J.-S.L.; funding acquisition, W.-C.L.; methodology, J.-S.L.; project administration, Y.-Y.C.; resources, C.-C.W. and H.Y.-L.S.; software, J.-S.L.; supervision, H.Y.-L.S.; validation, K.-M.R. and M.-C.H.; visualization, C.-C.W.; writing-original draft, C.-C.W.; and writing-review and editing, H.Y.-L.S.

Funding: The study was supported in part by a grant from Chang Gung Memorial Hospital, Kaohsiung, Taiwan. (CMRPG8F0581).

Acknowledgments: The study was based and generated on data from the National Health Insurance Research Database, provided by the Bureau of National Health Insurance and Ministry of Health and Welfare, managed by National Health Research Institutes. The interpretation and conclusions contained herein do not represent those of the aforementioned offices. The study was supported in part by a grant from Chang Gung Memorial Hospital, Kaohsiung, Taiwan. (CMRPG8F0581).

Conflicts of Interest: The authors declare no conflicts of interest. The funders had no role in the study.

\section{References}

1. Siegal, R.L.; Miller, K.D.; Jemal, A. Cancer Statics. CA Cancer J. Clin. 2016, 66, 7-30.

2. Chiang, C.J.; Lo, W.C.; Yang, Y.W.; You, S.L.; Chen, C.J.; Lai, M.S. Incidence and survival of adult cancer patients in Taiwan, 2002-2012. J. Formos. Med. Assoc. 2016, 115, 1076-1088. [CrossRef] [PubMed]

3. Hanna, N.; Johnson, D.; Temin, S.; Baker, S., Jr.; Brahmer, J.; Ellis, P.M.; Giaccone, G.; Hesketh, P.J.; Jaiyesimi, I.; Leighl, N.B.; et al. Systemic therapy for stage IV non-small-cell lung cancer: American Society of Clinical Oncology Clinical Practice Guideline update. J. Clin. Oncol. 2017, 35, 3484-3515. [CrossRef] [PubMed]

4. Rosell, R.; Carcereny, E.; Gervais, R.; Vergnenegre, A.; Massuti, B.; Felip, E.; Palmero, R.; Garcia-Gomez, R.; Pallares, C.; Sanchez, J.M.; et al. Erlotinib versus standard chemotherapy as first-line treatment for European patients with advanced EGFR mutation-positive non-small-cell lung cancer (EURTAC): A multicentre, open-label, randomised phase 3 trial. Lancet Oncol. 2012, 13, 239-246. [CrossRef]

5. Mok, T.S.; Wu, Y.L.; Thongprasert, S.; Yang, C.H.; Chu, D.T.; Saijo, N.; Sunpaweravong, P.; Han, B.; Margono, B.; Ichinose, Y.; et al. Gefitinib or carboplatin-paclitaxel in pulmonary adenocarcinoma. N. Engl. J. Med. 2009, 361, 947-957. [CrossRef] [PubMed]

6. Yang, J.C.H.; Wu, Y.L.; Schuler, M.; Sebastian, M.; Popat, S.; Yamamoto, N.; Zhou, C.; Hu, C.P.; O’Byrne, K.; Feng, J.; et al. Afatinib versus cisplatin-based chemotherapy for EGFR mutation-positive lung adenocarcinoma (LUX-Lung 3 and LUX-Lung 6): Analysis of overall survival data from two randomised, phase 3 trials. Lancet Oncol. 2015, 16, 141-151. [CrossRef]

7. Lee, C.K.; Davies, L.; Wu, Y.L.; Mitsudomi, T.; Inoue, A.; Rosell, R.; Zhou, C.; Nakagawa, K.; Thongprasert, S.; Fukuoka, M.; et al. Gefitinib or Erlotinib vs. Chemotherapy for EGFR Mutation-Positive Lung Cancer: Individual Patient Data Meta-Analysis of Overall Survival. J. Natl. Cancer Inst. 2017, 109, 1883-1891. [CrossRef]

8. Alexander, M.; Wolfe, R.; Ball, D.; Conron, M.; Stirling, R.G.; Solomon, B.; MacManus, M.; Officer, A.; Karnam, S.; Burbury, K.; et al. Lung cancer prognostic index: A risk score to predict overall survival after the diagnosis of non-small-cell lung cancer. Br. J. Cancer 2017, 117, 744-751. [CrossRef]

9. Islam, K.M.; Jiang, X.; Anggondowati, T.; Lin, G.; Ganti, A.K. Comorbidity and Survival in Lung Cancer Patients. Cancer Epidemiol. Biomark. Prev. 2015, 24, 1079-1085. [CrossRef]

10. Young, R.P.; Hopkins, R.J.; Christmas, T.; Black, P.N.; Metcalf, P.; Gamble, G.D. COPD prevalence is increased in lung cancer, independent of age, sex and smoking history. Eur. Respir. J. 2009, 34, 380-386. [CrossRef]

11. Ytterstad, E.; Moe, P.C.; Hjalmarsen, A. COPD in primary lung cancer patients: Prevalence and mortality. Int. J. Chron. Obstruct. Pulmon. Dis. 2016, 11, 625-636. [CrossRef] [PubMed]

12. Dy, S.M.; Sharkey, P.; Herbert, R.; Haddad, K.; Wu, A.W. Comorbid illnesses and health care utilization among Medicare beneficiaries with lung cancer. Crit. Rev. Oncol. Hematol. 2006, 59, 218-225. [CrossRef] [PubMed]

13. Gao, Y.H.; Guan, W.J.; Liu, Q.; Wang, H.Q.; Zhu, Y.N.; Chen, R.C.; Zhang, G.J. Impact of COPD and emphysema on survival of patients with lung cancer: A meta-analysis of observational studies. Respirology 2016, 21, 269-279. [CrossRef] [PubMed] 
14. Omote, N.; Hashimoto, N.; Morise, M.; Sakamoto, K.; Miyazaki, S.; Ando, A.; Nakahara, Y.; Hasegawa, Y. Impact of mild to moderate COPD on feasibility and prognosis in non-small cell lung cancer patients who received chemotherapy. Int. J. Chron. Obstruct. Pulmon. Dis. 2017, 12, 3541-3547. [CrossRef] [PubMed]

15. Izquierdo, J.L.; Resano, P.; El Hachem, A.; Graziani, D.; Almonacid, C.; Sanchez, I.M. Impact of COPD in patients with lung cancer and advanced disease treated with chemotherapy and/or tyrosine kinase inhibitors. Int. J. Chron. Obstruct. Pulmon. Dis. 2014, 9, 1053-1058. [CrossRef]

16. Mouronte-Roibas, C.; Leiro-Fernandez, V.; Fernandez-Villar, A.; Botana-Rial, M.; Ramos-Hernandez, C.; Ruano-Ravina, A. COPD, emphysema and the onset of lung cancer. A systematic review. Cancer Lett. 2016, 382, 240-244. [CrossRef]

17. Kiri, V.A.; Soriano, J.B.; Visick, G.; Fabbri, L.M. Recent trends in lung cancer and its association with COPD: An analysis using the UK GP Research Database. Prim. Care Respir. J. 2010, 19, 57-61. [CrossRef]

18. Tan, L.E.; Razak, A.M.; Lim, C.S. Association of chronic obstructive pulmonary disease and postresection lung cancer survival: A systematic review and meta-analysis. J. Investig. Med. 2017, 65, 342-352. [CrossRef]

19. Parrón Collar, D.; Pazos Guerra, M.; Rodriguez, P.; Gotera, C.; Mahillo-Fenandez, I.; Peces-Barba, G.; Seijo, L.M. COPD is commonly underdiagnosed in patients with lung cancer: Results from the RECOIL study (retrospective study of COPD infradiagnosis in lung cancer). Int. J. Chron. Obstruct. Pulmon. Dis. 2017, 12, 1033-1038. [CrossRef]

20. Sekine, Y.; Yamada, Y.; Chiyo, M.; Iwata, T.; Nakajima, T.; Yasufuku, K.; Suzuki, M.; Fujisawa, T. Association of chronic obstructive pulmonary disease and tumor recurrence in patients with stage IA lung cancer after complete resection. Ann. Thorac. Surg. 2007, 84, 946-950. [CrossRef]

21. Tokumo, M.; Toyooka, S.; Kiura, K.; Shigematsu, H.; Tomii, K.; Aoe, M.; Ichimura, K.; Tsuda, T.; Yano, M.; Tsukuda, K.; et al. The relationship between epidermal growth factor receptor mutations and clinicopathologic features in Non-small cell lung cancers. Clin. Cancer Res. 2005, 11, 1167-1173. [CrossRef]

22. Lim, J.U.; Yeo, C.D.; Rhee, C.K.; Kim, Y.H.; Park, C.K.; Kim, J.S.; Kim, J.W.; Lee, S.H.; Kim, S.J.; Yoon, H.K.; et al. Chronic Obstructive Pulmonary Disease-Related Non-Small-Cell Lung Cancer Exhibits a Low Prevalence of EGFR and ALK Driver Mutations. PLoS ONE 2015, 10, e0142306. [CrossRef] [PubMed]

23. Takeda, K.; Yamasaki, A.; Igishi, T.; Kawasaki, Y.; Ito-Nishii, S.; Izumi, H.; Sakamoto, T.; Tonge, H.; Kodani, M.; Makino, H.; et al. Frequency of Epidermal Growth Factor Receptor Mutation in Smokers with Lung Cancer without Pulmonary Emphysema. Anticancer Res. 2017, 37, 765-771. [CrossRef] [PubMed]

24. Douillard, J.Y.; Shepherd, F.A.; Hirsh, V.; Harris, P.L.; Okimoto, R.A.; Brannigan, B.W.; Sgroi, D.C.; Muir, B.; Riemenschneider, M.J.; Iacona, R.B.; et al. Epidermal growth factor receptor mutations and gene amplification in non-small-cell lung cancer: Molecular analysis of the IDEAL/INTACT gefitinib trials. J. Clin. Oncol. 2005, 23, 8081-8092.

25. Xiao, D.; Li, F.; Pan, H.; Liang, H.; Wu, K.; He, J. Integrative analysis of genomic sequencing data reveals higher prevalence of LRP1B mutations in lung adenocarcinoma patients with COPD. Sci. Rep. 2017, 7, 2121. [CrossRef] [PubMed]

26. Beer, A.G.; Zenzmaier, C.; Schreinlechner, M.; Haas, J.; Dietrich, M.F.; Herz, J.; Marschang, P. Expression of a recombinant full-length LRP1B receptor in human non-small cell lung cancer cells confirms the postulated growth-suppressing function of this large LDL receptor family member. Oncotarget 2016, 7, 68721-68733. [CrossRef] [PubMed]

27. Ni, S.; Hu, J.; Duan, Y.; Shi, Y.; Li, R.; Wu, H.; Qu, Y.; Li, Y. Down expression of LRP1B promotes cell migration via RhoA/Cdc42 pathway and actin cytoskeleton remodeling in renal cell cancer. Cancer Sci. 2013, 104, 817-825. [CrossRef]

28. Tabouret, E.; Labussiere, M.; Alentorn, A.; Schmitt, Y.; Marie, Y.; Sanson, M. LRP1B deletion is associated with poor outcome for glioblastoma patients. J. Neurol. Sci. 2015, 358, 440-443. [CrossRef]

29. De-Torres, J.P.; Sanchez-Salcedo, P.; Bastarrika, G.; Alcaide, A.B.; Pio, R.; Pajares, M.J.; Campo, A.; Berto, J.; Montuenga, L.; Del Mar Ocon, M.; et al. Telomere length, COPD and emphysema as risk factors for lung cancer. Eur. Respir. J. 2017, 49, 1601521. [CrossRef]

30. Kim, E.S.; Ye, Y.; Vaporciyan, A.A.; Xing, J.; Huang, M.; Gu, J.; Roth, J.A.; Lippman, S.M.; Wu, X. Telomere length and recurrence risk after curative resection in patients with early-stage non-small-cell lung cancer: A prospective cohort study. J. Thorac. Oncol. 2015, 10, 302-308. [CrossRef]

31. Thom, S.R.; Ma, M.; Bhopale, V.M.; Zhou, C.; Mao, L. Oxidative stress mediated by nitrogen at elevated pressure inhibits non-small cell lung cancer growth. Exp. Lung Res. 2017, 43, 175-180. [CrossRef] [PubMed] 
32. Bozinovski, S.; Vlanhos, R.; Anthony, D.; McQualter, J.; Anderson, G.; Irving, L.; Steinfort, D. COPD and squamous cell lung cancer: Aberrant inflammation and immunity is the common link. Br. J. Pharmacol. 2016, 173, 635-648. [CrossRef] [PubMed]

33. We, C.C.; Hsu, H.Y.; Liu, H.P.; Chang, J.W.; Chen, Y.T.; Hsieh, W.Y.; Hsieh, J.J.; Hsieh, M.S.; Chen, Y.R.; Huang, S.F. Reversed mutation rates of KRAS and EGFR genes in adenocarcinoma of the lung in Taiwan and their implications. Cancer 2008, 113, 3199-3208.

34. Midha, A.; Dearden, S.; McCormack, R. EGFR mutation incidence in non-small-cell lung cancer of adenocarcinoma histology: A systematic review and global map by ethnicity (mutMapII). Am. J. Cancer Res. 2015, 5, 2892-2911. [PubMed]

35. Shi, Y.; Au, J.S.K.; Thongprasert, S.; Srinivasan, S.; Tsai, C.M.; Khoa, M.T.; Heeroma, K.; Itoh, Y.; Cornelio, G.; Yang, P.C. A Prospective, Molecular Epidemiology Study of EGFR Mutations in Asian Patients with Advanced Non-Small-Cell Lung Cancer of Adenocarcinoma Histology (PIONEER). J. Thorac. Oncol. 2014, 9, 154-162. [CrossRef]

(C) 2019 by the authors. Licensee MDPI, Basel, Switzerland. This article is an open access article distributed under the terms and conditions of the Creative Commons Attribution (CC BY) license (http://creativecommons.org/licenses/by/4.0/). 